This item was submitted to Loughborough's Research Repository by the author.

Items in Figshare are protected by copyright, with all rights reserved, unless otherwise indicated.

\title{
Contributions of twisting techniques used in backward somersaults with one twist
}

PLEASE CITE THE PUBLISHED VERSION

http://journals.humankinetics.com/AcuCustom/SiteName/Documents/Documentltem/2373.pdf

\section{PUBLISHER}

(C) Human Kinetics

VERSION

AM (Accepted Manuscript)

LICENCE

CC BY-NC-ND 4.0

\section{REPOSITORY RECORD}

Yeadon, Maurice R., and David G. Kerwin. 2019. "Contributions of Twisting Techniques Used in Backward Somersaults with One Twist”. figshare. https://hdl.handle.net/2134/6237. 
This item was submitted to Loughborough's Institutional Repository (https://dspace.lboro.ac.uk/) by the author and is made available under the following Creative Commons Licence conditions.

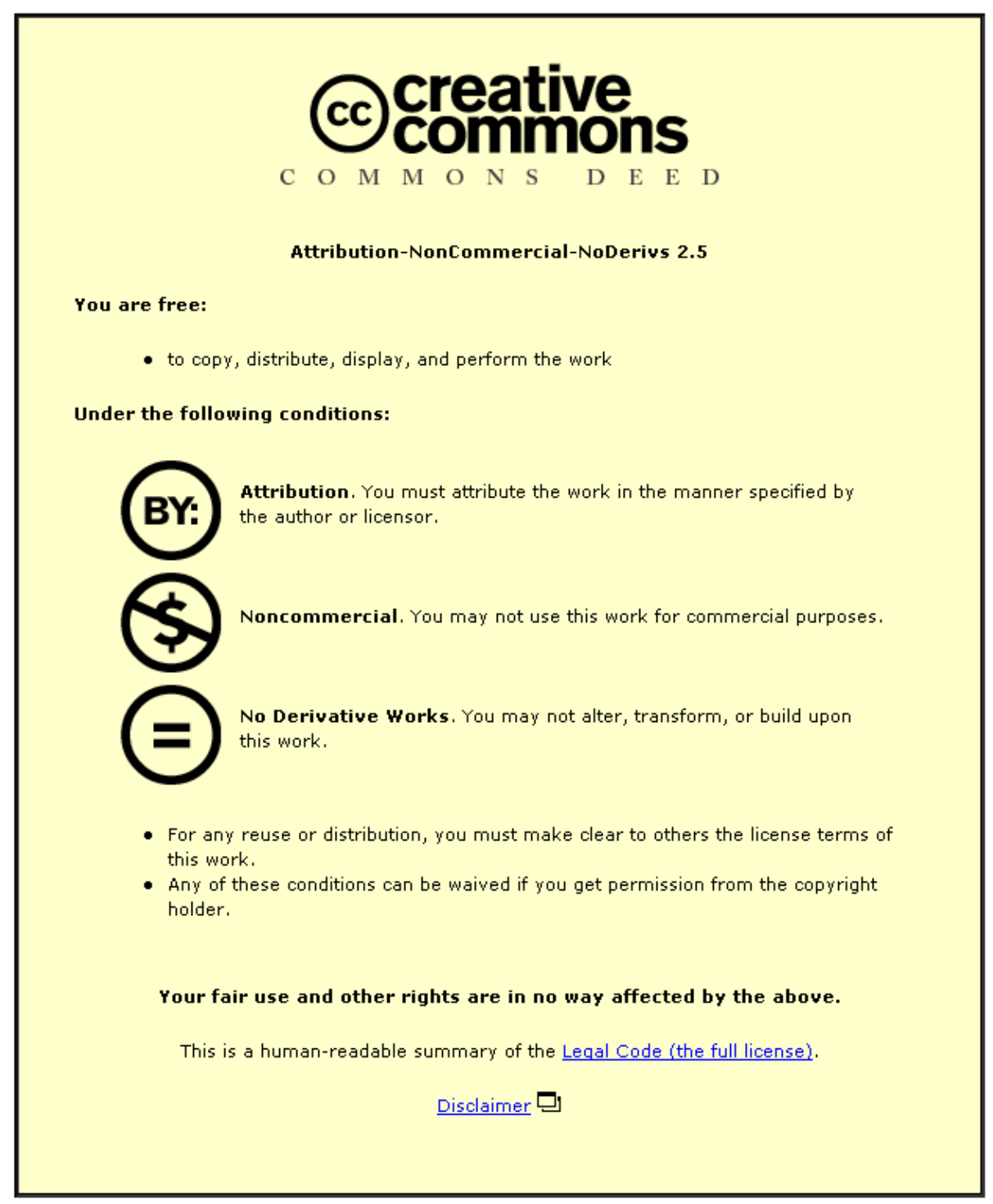

For the full text of this licence, please go to: http://creativecommons.org/licenses/by-nc-nd/2.5/ 


\title{
Contributions of twisting techniques used in backward somersaults with one twist
}

\author{
Maurice R. Yeadon and David G. Kerwin \\ Department of Sports Science, Loughborough University, Leics. LE11 3TU, UK
}

\begin{abstract}
At the 1996 Atlanta Olympic Games, 18 single somersaults with one twist in the women's compulsory floor exercises were recorded using two video cameras. An 11 segment computer simulation model was used to analyse the twisting techniques used. It was found that counter-rotation techniques accounted for less than one third of the twist for all gymnasts indicating that the production of twist was mainly a consequence of the angular momentum and a non-zero tilt angle. Contributions to the tilt angle reached at the mid-twist position were used as measures of the twisting potential of various techniques. Contact techniques accounted for $30 \%$ of the tilt produced, the remainder being produced using aerial techniques which primarily comprised a symmetrical lowering of the arms together with minor contributions from asymmetrical arm and hip movements. There was no evidence of a difference in technique between the highest and lowest scoring competitors.
\end{abstract}

Keywords: twisting, somersault, simulation, floor, tumbling, gymnastics

\section{Introduction}

The women's floor exercise in Artistic Gymnastics combines dance movements with tumbling skills. A typical competition exercise includes three or four tumbling sequences involving single or double somersaults with and without twist. Several coaching articles state that the twist in a single somersault on floor is initiated during the contact phase (Boone, 1974; Smith, 1980; Smith, 1982; Dowdell, 1988). As a consequence many coaches instruct their gymnasts to start the twist during the takeoff phase. Cinematographic studies of single somersaults with one or two twists on floor have concluded that twisting started while the gymnasts were still in contact with the floor (Wiley, 1964; Seidel, 1976; AlHaroun, 1980). Moore (1951) progressively restricted a trampolinist's freedom of movement using plaster casts and splints. He found that full twisting somersaults could still be performed even when relative movements between body segments was almost totally restricted. The amount of twist observed during the contact phase increased as more parts of the body were immobilized. While this study indicates that it is certainly possible to initiate the twist during the contact phase, it also suggests that there may be a contribution from relative movements of the limbs during the aerial phase.

Aerial twist is used by cats when dropped in an inverted position (McDonald, 1960; Frederick, 1971). Experiments have shown humans can produce up to one twist after takeoff using a hula-hoop movement of the hips (McDonald, 1961). Van Gheluwe and Duquet (1977) found some evidence of this type of hip movement during full twisting backward somersaults on floor. This counter-rotation technique should be capable of making substantial contributions to somersaults with one twist but it is likely to make relatively smaller contributions when the number of twists is large since around one cycle of hip movement is required for each half twist of the body (Yeadon, 1993c).

A sustained aerial twist may be initiated during a plain somersault by tilting the longitudinal axis of the body away from the plane perpendicular to the angular momentum vector (Batterman, 1974). In theory this may be accomplished using asymmetrical movements of the arms (Frolich, 1979) or asymmetrical movements of the chest or hips (Yeadon, 1993c). Van Gheluwe (1981) used a six segment computer simulation model to evaluate filmed performances of three full twisting somersaults on a trampoline. By using simulations based on modifications of the film data it was shown that asymmetrical arm movements during the aerial phase were responsible for the production of twist. 
A method of partitioning the contributions of twisting techniques was developed by Yeadon (1993d) who used the angle of tilt as a measure of the potential twist rate. Using an 11 segment simulation model Yeadon et al. (1990b) showed that for eight single somersault dismounts from high bar with one twist, the majority of the tilt arose from asymmetrical movements during the aerial phase. For eight double somersault dismounts from high bar with one or two twists it was found that the contact contribution was larger when there was more twist in the first somersault (Yeadon, 1997).

It appears that twisting in single somersaults on floor starts during the contact phase although it is unclear whether aerial techniques make substantial contributions. Since technique can vary with ability (Sanders, 1995) it may be the case that elite gymnasts have more of an aerial contribution compared with less skilled competitors. It is the aim of this study to determine the contributions of contact and aerial techniques to the production of twist and to investigate whether there is a difference between the highest scoring and the lowest scoring Olympic competitors.

\section{Methods}

\section{Data Collection}

Five days prior to the women's team competition, the training sessions on floor were observed in order to determine which part of the floor should be calibrated in order to obtain as many full twisting somersaults as possible. It was concluded that one corner of the floor area was used by the majority of the competitors for the full twisting somersault in the compulsory floor exercise. The day before the competition a calibration pole, with spheres of diameter $0.08 \mathrm{~m}$ set at heights of $0.30 \mathrm{~m}, 1.20 \mathrm{~m}, 2.19 \mathrm{~m}$ and $3.14 \mathrm{~m}$, was placed in eight measured locations encompassing the last five metres of one diagonal of the floor area. Video recordings were made of the calibration pole in each position.

The compulsory floor exercises of the Women's Artistic Gymnastics team competition at the 1996 Atlanta Olympic Games were video-recorded using a Sony Hi 8 camcorder (CCD-VX1E) and a Sony digital camcorder (DCR-VX11000E). The cameras were placed at the ends of a $27 \mathrm{~m}$ platform $10 \mathrm{~m}$ above and $35 \mathrm{~m}$ back from the competition floor area. The cameras used shutter times of $1 / 300 \mathrm{~s}$ and recorded at 50 fields per second providing around 50 fields for the aerial phase of each twisting somersault.

\section{Data Processing}

Of the 94 competitors 46 performed the full twisting somersault of their compulsory floor exercise in the corner that had been calibrated. Two groups of nine competitors were selected for analysis from these 46. Group A comprised the nine highest scoring competitors (9.70 - 9.85) while Group B comprised the nine lowest scoring competitors $(8.61-9.26)$.

The images of the calibration pole were digitized for each camera view. Camera calibration was carried out using a 12 parameter Direct Linear Transformation procedure (Karara, 1980) and unbiased estimates of reconstruction accuracy were determined.

The two views of the aerial phase of each dismount were digitized. In each video field of the aerial phase the wrist, elbow, shoulder, hip, knee and ankle centers were digitized, giving 12 landmarks in total. The head was assumed to be in normal alignment and the ankles were assumed to be plantar-flexed. The three-dimensional coordinates of the joint centers were reconstructed by finding a least squares solution to four planes defined by the DLT equations. An estimate of the reconstruction error was calculated as an unbiased root mean square distance from the four planes.

Interpolating quintic splines (Wood and Jennings, 1979) were fitted to the digitized coordinate data to allow the estimation of data values at times in between those of the video fields. In order to determine the synchronization offset between the data obtained from the two cameras, different offsets at intervals of $0.001 \mathrm{~s}$ were used. For each time offset the corresponding root mean square distance of the 12 joint centers from the four DLT planes over all video fields was calculated. The synchronization offset that minimised the root mean square distance was used.

The body was modelled using four arm segments, four leg segments and three torso segments. The orientation of the body was defined by angles corresponding to somersault, apparent tilt, and twist where somersault is the rotation about a horizontal axis, twist is the rotation about the longitudinal body axis and apparent tilt is the angle between the longitudinal axis and the vertical somersault plane (Figure 
1). The longitudinal axis was defined as a line through the mass center parallel to the line joining the midpoints of the knee centers and shoulder centers. Body configuration was specified using 14 angles to describe the relative orientations of the body segments. These 14 angles comprised angles of flexion, abduction and rotation at each shoulder joint, flexion angles at each elbow, tilt and torsion of the chest relative to the abdomen, one abduction angle for both thighs, one flexion angle for both knee joints, one flexion angle for the hip joints and the joint at the junction of the pelvis and abdomen, and one "hula" angle indicating whether flexion was forwards, backwards or sideways (Yeadon, 1990c).

The time histories of the orientation and configuration angles were determined from the threedimensional coordinates of the digitized joint centers (Yeadon, 1990a). A pseudo data set was derived from the digitised data using the mean value of the previous field and the following field. The differences between the angle values and the corresponding pseudo angle values were used to provide reliability estimates of the angle data for use in fitting quintic splines. A combination of global and local reliability estimates were used as described in Yeadon (1990a) to fit quintic splines (Wood and Jennings, 1979) to the time histories of the angles.

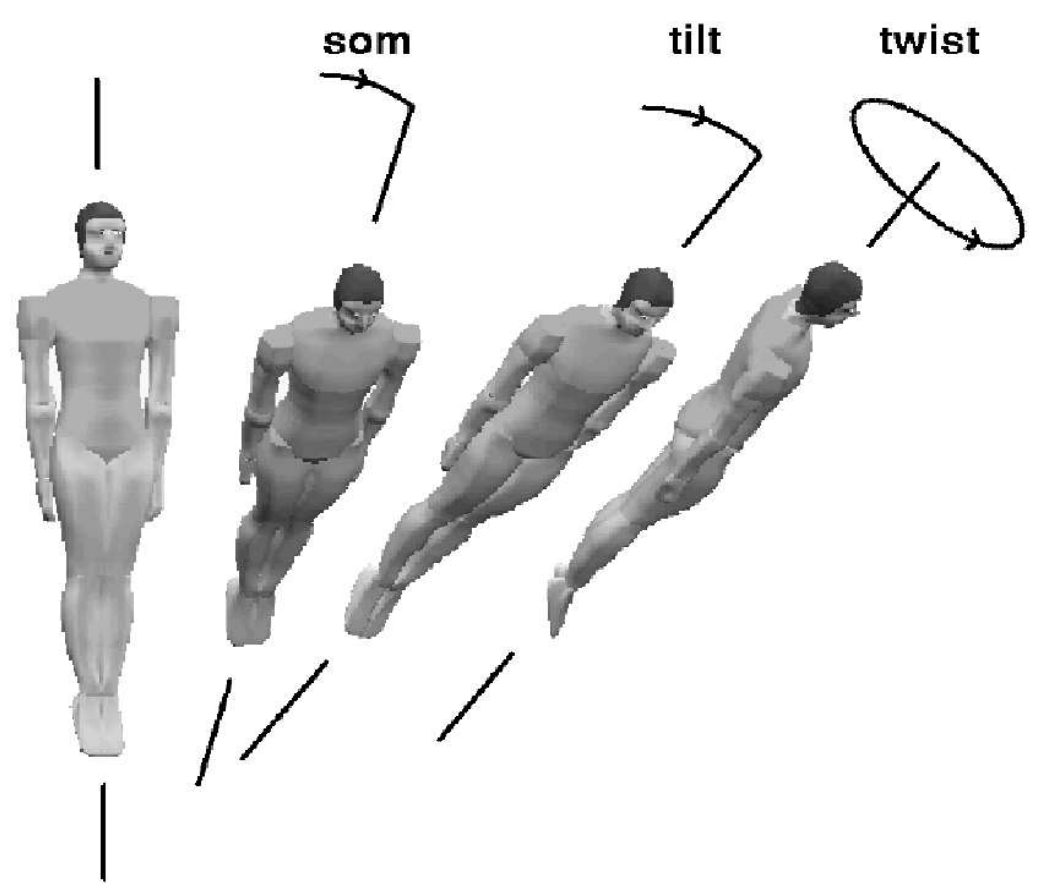

Figure 1: Angles of somersault, apparent tilt, and twist.

Anthropometric measurements were taken on six members of the British Gymnastics Squad and were averaged to produce a mean anthropometric data set. The three-dimensional data of the joint centers obtained from the digitized performances of the 18 gymnasts were used to determine mean segment lengths and shoulder and hip width for each gymnast. These measurements were used to produce an individualized anthropometric data set from the mean anthropometric data. Lengths were scaled using the appropriate segment length, the upper torso and arm perimeters and widths were scaled in proportion to the shoulder width, and the lower torso and leg perimeters and widths were scaled in proportion to the hip width. Segmental masses, mass center locations, link lengths and moments of inertia were determined for each individualized anthropometric data set using the mathematical inertia model of Yeadon (1990b).

\section{Data Analysis}

An 11 segment model was used to determine the angular momentum vector during each of the 18 full twisting somersaults using the time histories of the 17 orientation and configuration angles (Yeadon, 1990c). The mean values of the calculated angular momentum components and the time histories of the 14 configurational angles, together with the initial values of the three orientation angles and the 
segmental inertia parameters, were used as input to an 11 segment simulation model (Yeadon et al., 1990a).

The angles of somersault, apparent tilt, and twist in each simulation were compared with the corresponding angles obtained from the three-dimensional video analysis to evaluate the performance of the simulation model. Subsequently the shoulder and hip width estimates were modified by up to $0.02 \mathrm{~m}$ to see what improvement in agreement between simulation and video resulted from the modified inertia parameters. These modified parameters were used in the subsequent simulations.

In order to assess the importance of counter-rotation techniques in the production of twist, modified simulations were carried out with the total angular momentum set to zero. The final twist angle in the modified simulation was compared with the final twist angle in the original movement.

The simulation model was used to determine the direction of the principal axis corresponding to minimum moment of inertia and to calculate the angle of tilt between this axis and the invariable plane perpendicular to the angular momentum vector. This angle of tilt is a kinetic variable in the sense that it describes an aspect of the mechanics of the system. It should be distinguished from the angle of apparent tilt which is a kinematic variable describing orientation relative to the vertical somersault plane (Yeadon, $1993 \mathrm{~b})$.

The relationship between the twist rate p, the tilt angle $\theta$ and the somersault rate $\Omega$ for an axially symmetric rigid body with principal moments of inertia $A, A$ and $C$ (where $A>C$ ) is given in Yeadon (1993a) as:

$$
p=\Omega[A / C-1] \sin \theta
$$

Thus the angle of tilt between the minimum principal axis of the body and the invariable plane perpendicular to the angular momentum vector may be used to give the twist per somersault rate for a given body configuration, providing that the two large principal moments of inertia are approximately equal. Since the angle of tilt is approximately proportional to the twist rate for a given body configuration, it may therefore be used as a measure of the twisting potential present, independent of the actual configuration adopted. In other words there is a maximum twist rate associated with a particular tilt angle corresponding to a straight body configuration with arms and legs adducted. The actual twist angle will of course be dependent upon the body configuration used and the time at which twisting is initiated. The advantage of using the tilt angle (or potential twist rate) as a measure is that the contributions of various techniques are additive whereas this is not the case if the twist angle is used.

Twisting techniques are defined as contact or aerial techniques, depending on whether twisting is initiated during contact with the floor or during the aerial phase (Yeadon, 1993b, 1993c). The initial value of the tilt angle at takeoff from the floor is a measure of twisting initiated during contact with the floor. The subsequent increase in the tilt angle during the somersault gives the contribution of aerial twisting techniques to the total tilt produced.

In order to partition the aerial contribution to the tilt angle into a symmetry contribution and contributions associated with left-right asymmetries of arms, chest and hips, the configurational angles obtained from video were modified. These modifications comprised the systematic removal of asymmetries of the arms, chest and hips relative to the sagittal plane. Arm movement was made symmetrical by having the left arm mirror the recorded movement of the right arm and vice-versa, chest torsion relative to the abdomen was eliminated, and lateral flexion was removed from the movement of the abdomen and legs relative to the pelvis by only permitting flexion parallel to the sagittal plane. The systematic removal of arm, chest and hip asymmetries provided 12 configuration sequences ranging from the original movement to a sequence with complete left-right configurational symmetry (Table 1). The tilt angle produced at the time corresponding to the half twist position in the simulation of the original movement was determined from simulations based upon the modified configurations. The tilt contribution due to symmetry was calculated as the average amount that the tilt angle rose above the initial value in the two simulations in which left-right configurational symmetry was maintained $([0,0,0]$ and $[2,0,0]$ in Table 1$)$.

The tilt associated with asymmetrical arm movement could be calculated as the mean difference between the tilt values for the simulations of complete symmetry $([0,0,0]$ and $[2,0,0])$ and of symmetrical chest and hips $([1,0,0])$. It could also be calculated as the mean difference between the tilt values for the simulations corresponding to arm symmetry $([0,1,1]$ and $[2,1,1])$ and the original configuration $([1,1,1])$. The average of the tilt values obtained in these two ways was taken as the tilt contribution from asymmetrical arm movement. The other contributions to the tilt angle were calculated in a similar 
way as described in Yeadon (1993d). The total of the tilt contributions was compared with the actual tilt angle produced in the unmodified simulation as a test of the additivity of the contributions.

Table 1: 12 configurational asymmetries

\begin{tabular}{|l|l|l|l|l|l|l|l|l|l|l|l|l|}
\hline arm & 0 & 2 & 0 & 2 & 0 & 2 & 0 & 2 & 1 & 1 & 1 & 1 \\
\hline chest & 0 & 0 & 1 & 1 & 0 & 0 & 1 & 1 & 0 & 1 & 0 & 1 \\
\hline hip & 0 & 0 & 0 & 0 & 1 & 1 & 1 & 1 & 0 & 0 & 1 & 1 \\
\hline
\end{tabular}

Note: $1=$ asymmetry and $0,2=$ symmetry. For the arms 0 represents the left arm mirroring the original right arm movement while 2 represents the right arm mirroring the original left arm movement

The symmetry contribution to the tilt angle is partly a consequence of nutation whereby the tilt angle increases during the first quarter twist due to the difference in the moments of inertia about the lateral and frontal axes (Yeadon, 1993e). If the arms are wide during the first quarter twist this increase in the tilt angle can be several degrees. During the second quarter twist the process is reversed and the tilt angle decreases but the magnitude of this effect is small if the arms are brought close to the body.

A symmetry contribution can be also be produced by lowering the arms parallel to the sagittal plane at around the quarter twist position so that the body counter-rotates, increasing the tilt angle. In order to determine the contribution made by lowering the arms symmetrically, a modified symmetrical simulation was used in which the angular momentum was set to zero but the twist orientation was reset to the video value after each integration step. The tilt produced in this simulation was a result of the symmetrical lowering of the arms parallel to the sagittal plane.

\section{Results}

The reconstruction errors of the calibration markers were $0.0083 \mathrm{~m}$ along the floor diagonal, $0.0076 \mathrm{~m}$ laterally and $0.0034 \mathrm{~m}$ vertically. The horizontal errors were greater for the higher markers than for the lower markers, indicating that the vertical alignment of the pole was not consistent. Mean error estimates of the reliability of digitizing the body landmarks were found to lie between $0.010 \mathrm{~m}$ and $0.012 \mathrm{~m}$ for the 18 somersaults. The estimates of the three-dimensional reconstruction errors of body landmarks ranged from $0.007 \mathrm{~m}$ to $0.013 \mathrm{~m}$. The difference between the values of the orientation angles obtained from simulation and video were evaluated at the time corresponding to the half twist position in the original simulation. Mean deviations were $0.016 \mathrm{rev}$. for somersault, $1.4^{\circ}$ for apparent tilt and $0.043 \mathrm{rev}$. for twist. When inertia parameters were adjusted by changing the shoulder and hip width values by up to $0.02 \mathrm{~m}$ the mean deviations changed to $0.016 \mathrm{rev}$. for somersault, $1.2^{\circ}$ for apparent tilt and $0.021 \mathrm{rev}$. for twist. Figure 2 presents a typical comparison of video and simulation sequences for competitor 287. It can be seen that the differences between the two sequences increase as the simulation progresses. This is to be expected since the errors are cumulative due to the process of numerical integration inherent in a simulation.

The mean twist produced by counter-rotation when the angular momentum was set to zero was 0.16 revolutions for the high scoring group and 0.20 for the low scoring group (Table 2) although these values were not significantly different $(\mathrm{p}>0.05)$. Since this method produced less than one third of the twist for all 18 competitors, the production of twist was primarily a consequence of the angular momentum and a non-zero tilt angle. Competitor 331 produced 0.18 twists using counter-rotation of the hips. Figure 3 shows the original movement (upper sequence) together with the result of a modified simulation in which the body configuration was the same but the angular momentum was set to zero (lower sequence). In the fourth graphic of the lower sequence it can be seen that the body is flexed over the right hip indicating hip and leg circling to the right which resulted in the twist to the left.

Tilt contributions for the production of twist were evaluated for the time at which the original simulation reached the half twist position. These contributions are representative of the respective twist 

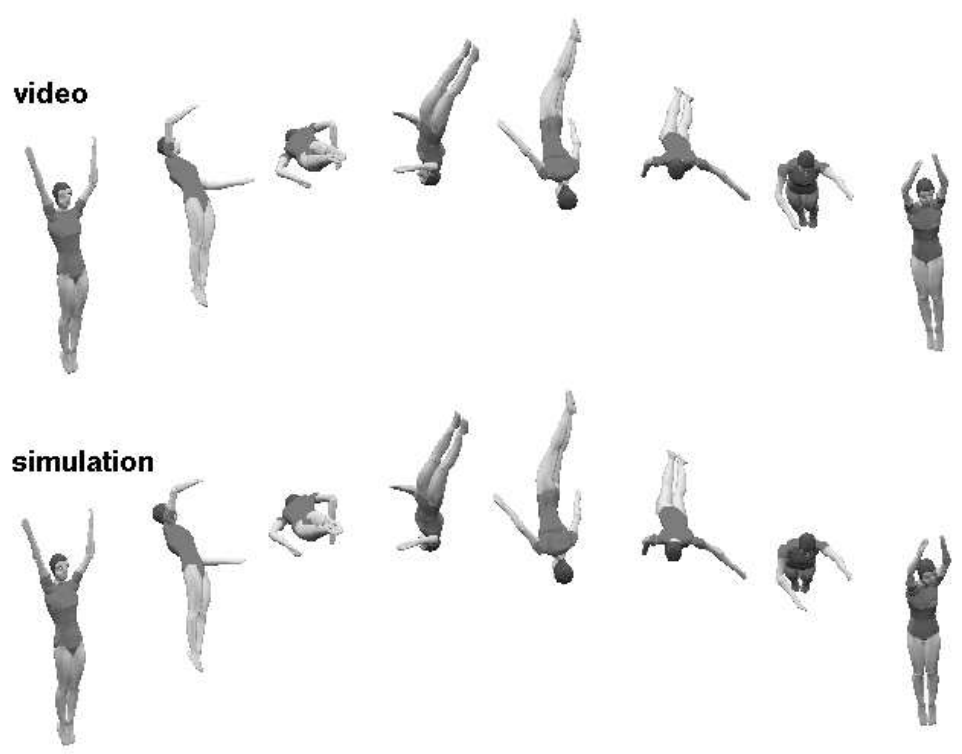

Figure 2: Comparison of video and simulation for competitor 287.

Table 2: Twist produced by counter-rotation techniques

\begin{tabular}{|l|l|l|l|l|l|}
\hline & \multicolumn{4}{|c|}{ simulation } & video \\
\hline & mean & SD & min & max & mean \\
\hline Group A & 0.16 & 0.05 & 0.09 & 0.22 & 0.98 \\
\hline Group B & 0.20 & 0.07 & 0.12 & 0.33 & 1.00 \\
\hline
\end{tabular}

Note: all twist values are in revolutions Group A : high scoring

Group B : low scoring

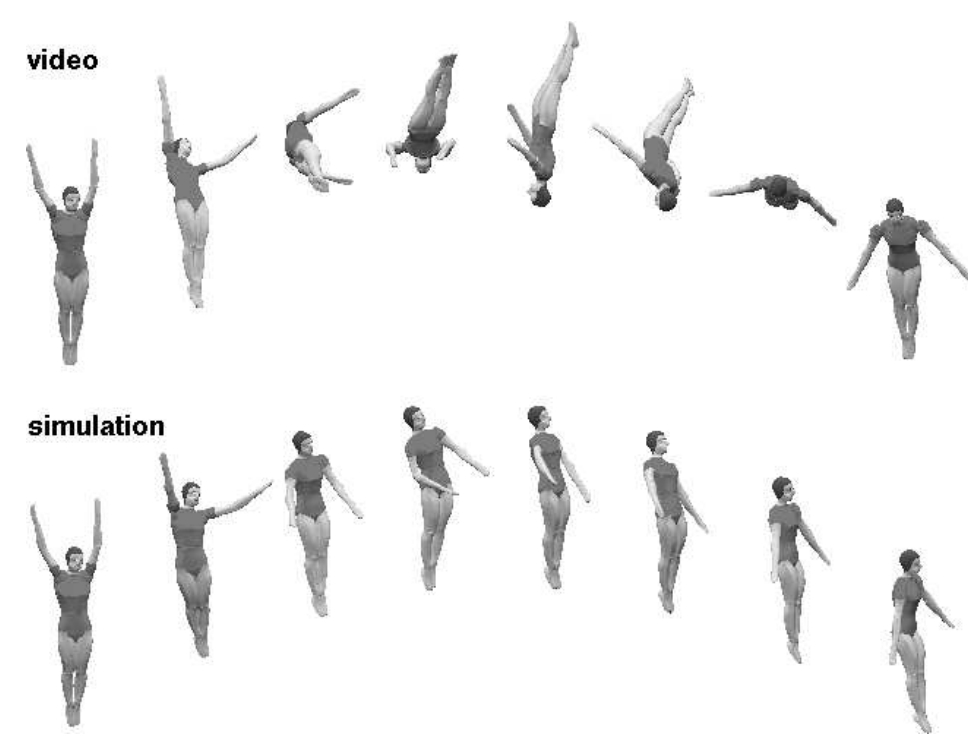

Figure 3: Graphics sequences of competitor 331. The upper sequence shows the original movement while the lower sequence shows the twist resulting from counter-rotation of the hips in a simulation with the angular momentum set to zero. 
Table 3: Contributions (in degrees) to the production of tilt by Groups A and B

\begin{tabular}{|l|c|c|c|c|c|c|c|}
\hline Group A & initial & symmetry & arms & chest & hips & total & actual \\
\hline 211 & 3 & 5 & 1 & 2 & 3 & 13 & 13 \\
\hline 287 & 2 & 3 & 2 & 1 & 0 & 9 & 8 \\
\hline 291 & 6 & 4 & 1 & -1 & -2 & 9 & 9 \\
\hline 299 & 3 & 4 & 0 & 0 & 0 & 6 & 6 \\
\hline 300 & 5 & 4 & 2 & -1 & 2 & 12 & 12 \\
\hline 310 & 1 & 4 & 2 & 1 & 1 & 9 & 9 \\
\hline 320 & 3 & 5 & 2 & 0 & 1 & 11 & 12 \\
\hline 331 & 2 & 2 & 0 & 2 & 6 & 11 & 11 \\
\hline 345 & 3 & 4 & 0 & 1 & 0 & 8 & 8 \\
\hline mean & $\mathbf{3}$ & $\mathbf{4}$ & $\mathbf{1}$ & $\mathbf{0}$ & $\mathbf{1}$ & $\mathbf{1 0}$ & $\mathbf{1 0}$ \\
\hline \multicolumn{7}{|r|}{} & \multicolumn{5}{|l|}{} & \multicolumn{2}{|c|}{} \\
\hline Group B & initial & symmetry & arms & chest & hips & total & actual \\
\hline 204 & 4 & 9 & 1 & 0 & -2 & 12 & 12 \\
\hline 208 & 2 & 1 & 2 & 3 & 1 & 8 & 8 \\
\hline 248 & 3 & 3 & 2 & 0 & 2 & 9 & 9 \\
\hline 249 & 3 & 7 & 2 & -1 & -2 & 9 & 9 \\
\hline 255 & 5 & 5 & 0 & 0 & 0 & 10 & 10 \\
\hline 273 & 5 & 4 & 0 & 0 & 0 & 9 & 9 \\
\hline 280 & 3 & 5 & 1 & 0 & 0 & 9 & 9 \\
\hline 284 & 3 & 2 & 0 & 0 & 1 & 5 & 6 \\
\hline 328 & 3 & 4 & 2 & 1 & 3 & 13 & 13 \\
\hline mean & $\mathbf{3}$ & $\mathbf{4}$ & $\mathbf{2}$ & $\mathbf{0}$ & $\mathbf{0}$ & $\mathbf{9}$ & $\mathbf{9}$ \\
\hline
\end{tabular}

Note: Contributions have been rounded to the nearest degree

rates rather than twist angles. Table 3 presents the contributions made to the tilt angle at the half twist position in each of the 18 dismounts. The contact contribution is the initial tilt angle between the longitudinal axis and the invariable plane perpendicular to the angular momentum vector evaluated at the moment of takeoff. The symmetry contribution indicates how much the tilt angle increases from its initial value during a simulation that enforces symmetry of the arms, chest and hips about the sagittal plane. The contributions listed under arms, chest and hips arise from asymmetrical arm movements, torsion of the chest, and lateral flexion at the hips.

For the 18 somersaults analyzed, the absolute deviations between the sum of the tilt contributions (as listed under "total") and the actual tilt value obtained from the unmodified simulation had a mean value of $0.2^{\circ}$ and a maximum value of $0.4^{\circ}$.

The tilt values at the half twist position ranged from $6^{\circ}$ to $13^{\circ}$ for both the high scoring group (A) and the low scoring group (B). Competitor 211 had a tilt angle of $13^{\circ}$ while competitor 299 had a tilt angle of $6^{\circ}$ (Figure 4). These gymnasts twisted in opposite directions so that their tilt angles are also in opposite directions. The initial tilt angles ranged from $6^{\circ}$ down to $1^{\circ}$ (competitors 291 and 310 in Figure (4). A high initial tilt angle indicates that the body is twisting at takeoff. Competitor 291 has completed almost one quarter twist at takeoff whereas competitor 310 appears to have not twisted at all at the time of takeoff.

Symmetry contributions ranged from $1^{\circ}$ to $9^{\circ}$ and comprised the largest contributions for more than half the gymnasts. Of the mean symmetry contribution of $4.1^{\circ}$ the lowering of the arms produced a mean $3.5^{\circ}$ tilt so that the mean contribution from nutation was $0.6^{\circ}$. This indicates that the lowering of the arms was typically parallel to the sagittal plane rather than to the sides. Competitor 204 had the largest symmetry contribution of $9.1^{\circ}$ of which $6.3^{\circ}$ was a result of lowering the arms as shown in Figure 5. The upper sequence shows the original movement while the lower sequence depicts a simulation with zero angular momentum in which the twist angle is reset to be the same as that of the original movement at the end of each integration step. 

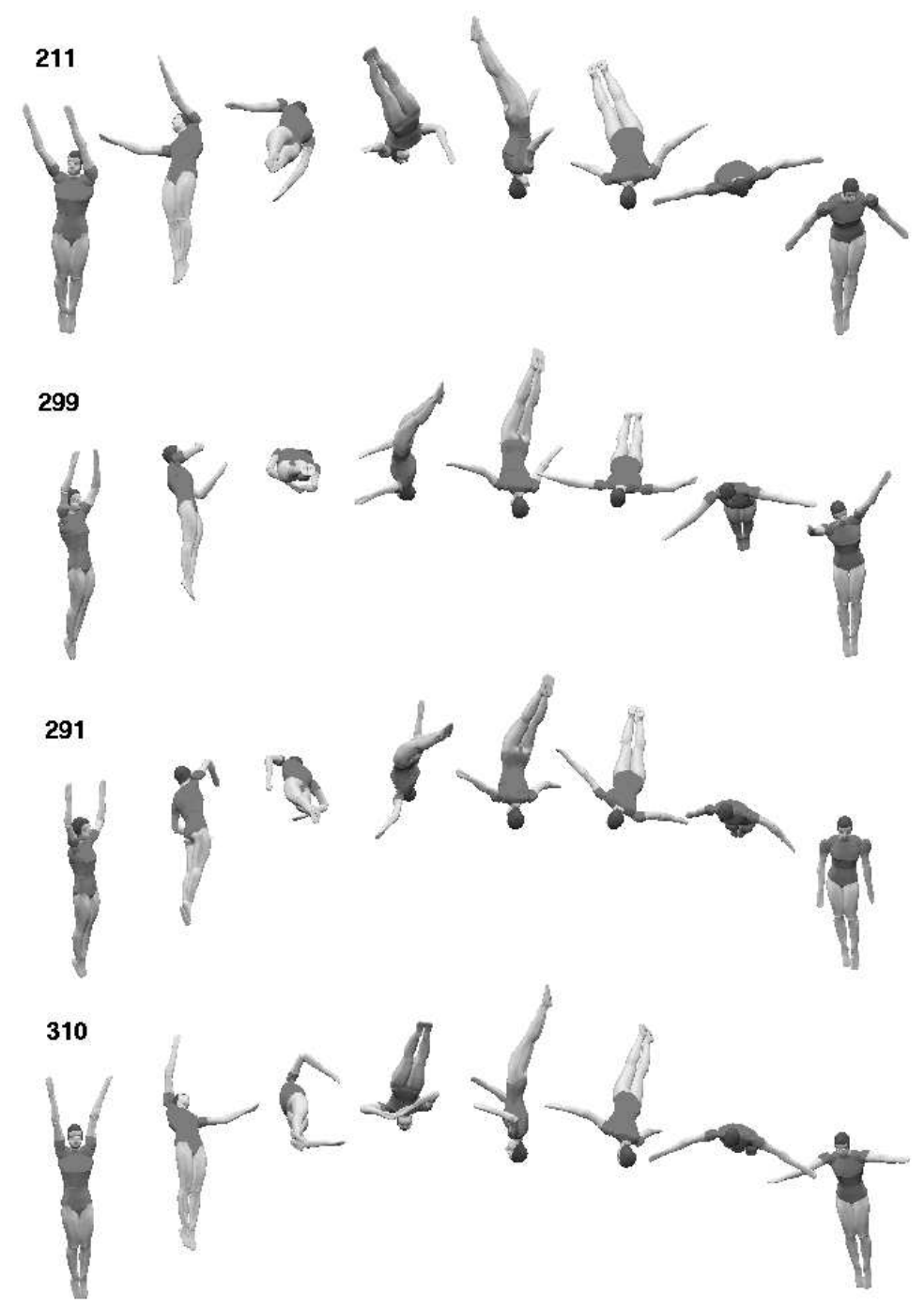

Figure 4: Graphics sequences showing large tilt (211), small tilt (299), contact twist (291) and aerial twist (310). 

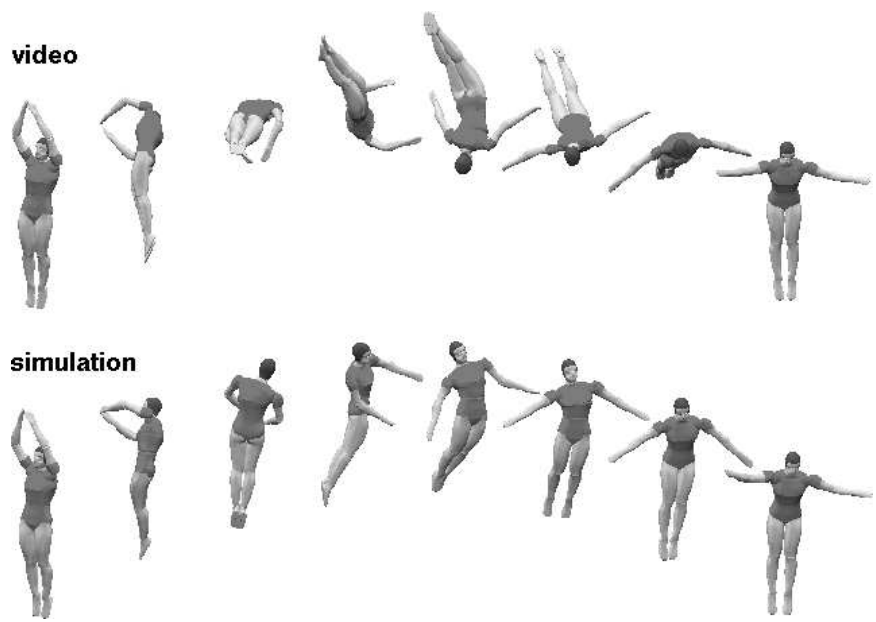

Figure 5: Graphics sequences of competitor 204. The upper sequence shows the original movement which had a large symmetry contribution while the lower sequence shows the tilt produced by lowering the arms.

\section{Discussion}

Although counter-rotation of the hips is capable of producing more than one twist in the aerial phase (McDonald, 1961), this technique was responsible for only $12 \%$ to $33 \%$ of the twist produced by each gymnast. This might be expected since, in order to score well, the body should be straight during the full twisting somersault while the hula-hoop counter-rotation technique requires the body to be flexed. It may be speculated that counter-rotation techniques will produce similar amounts of twist in single somersaults with two or three twists and consequently will play a relatively smaller role in the production of twist.

Since only a small portion of the total twist resulted from relative body segment movements under zero angular momentum conditions, the production of twist was primarily a result of the tilt of the longitudinal axis away from the plane perpendicular to the angular momentum vector. This tilt angle is a measure of the potential twist rate, irrespective of the body configuration actually adopted. The tilt angle is a more revealing measure of twisting technique than the twist angle since it may be partitioned into tilt contributions from contact and various aerial techniques. This is not possible using the final twist angle as a measure of performance since contact techniques cannot be separated from symmetry techniques. Additionally movements which remove the tilt and slow the twist in the original performance may increase the tilt angle and accelerate the twist in modified simulations so that the final twist angle will be unduly affected.

Both the highest scoring gymnasts (Group A) and the lowest scoring gymnasts (Group B) had a mean initial tilt angle of $3^{\circ}$. Since the initial tilt angle is a measure of the contribution of twisting initiated during the contact phase, the twisting started, typically, during the contact phase. This result is in agreement with the studies of Wiley, (1964), Seidel (1976) and Al-Haroun (1980). On the other hand, the contact techniques contributed only $3^{\circ}$ to the total tilt of $10^{\circ}$ for Group A and $9^{\circ}$ for Group B. Thus aerial techniques were responsible for the majority of the tilt produced and the consequent twisting. The mean symmetry contribution was $4^{\circ}$ for each group and was the largest aerial contributor to the tilt angle. The symmetry contribution was mainly a consequence of lowering the arms parallel to the sagittal plane during the first half twist. It should be noted that the tilt produced by lowering the arms will only be a positive contribution when the initial direction of somersault is backwards. Thus we would not expect the same result for forward somersaults with twist. The total of the mean contributions of arm, chest and hip asymmetries to the tilt angle was only $2^{\circ}$. This is in contrast to the asymmetrical contributions to full twisting dismounts from the high bar $\left(11^{\circ}\right.$ out of a total of $\left.15^{\circ}\right)$, full twisting double somersault dismounts from high bar $\left(5^{\circ}\right.$ out of $\left.8^{\circ}\right)$ and full twisting double somersault dismounts from rings $\left(7^{\circ}\right.$ out of $9^{\circ}$ ) as detailed in Yeadon et al. (1990b), Yeadon (1997) and Yeadon (1994). The reason for this difference might be that for dismounts from the high bar and rings the body is close to the horizontal 
at release so that only $\frac{3}{4}$ or $1 \frac{3}{4}$ somersaults are completed during the flight phase. In the case of a rigid body that has contact twist so that the initial tilt angle is non-zero, the apparent tilt angle away from the vertical will increase from an initial value of zero to a maximum after a half somersault and will return to zero after one somersault (Yeadon, 1993b). For a twisting somersault on floor this rigid body effect is not likely to cause any problems for landing since the somersault rotation is close to one revolution. For apparatus dismounts, however, the somersault rotation during the flight phase is not a whole number of somersaults. The rigid body effect will tend to lead to a tilted landing unless preventative action is taken during flight. Thus there are advantages to using aerial rather than contact techniques in dismounts from the high bar and rings. When the contact contribution is small the initial twist rate will be small so that any early symmetrical lowering of the arms will produce only small amounts of tilt. Since the symmetry contribution may be expected to be low when the contact contribution is low, the main contribution will be from asymmetrical arm, chest and hip movements in the aerial phase. Since these arguments do not apply to twisting somersaults from the floor the substantial contact and symmetry contributions to the tilt angle are understandable.

The tilt produced was more than ample for a full twist. For competitor 291 the body configuration in the aerial phase was modified so that the arms were held closer to the body. The modified simulation resulted in two revolutions of twist (Figure 6).

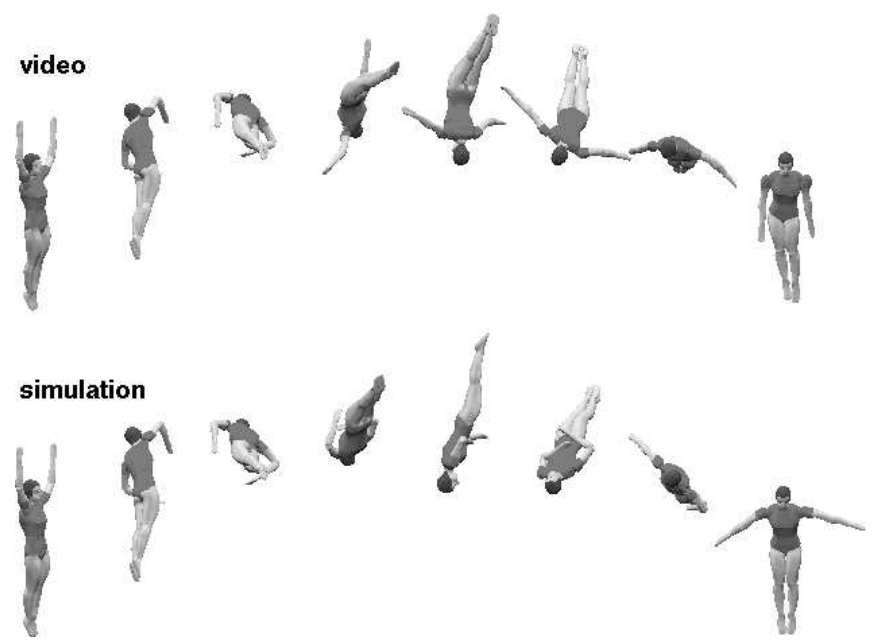

Figure 6: Graphics sequence of competitor 291. The upper sequence shows the original movement. In the lower sequence the body configuration has been modified so that the arms are held close to the body and two revolutions of twist are produced.

There was no evidence of a difference in technique between the highest and lowest scoring competitors. Indeed the mean contributions shown in Table 3 are remarkably similar for the two groups. The reason for this may be that all competitors were elite gymnasts and that the differences between the performances were technical rather than mechanical. There may be a mechanical difference in technique between elite and novice gymnasts where the difference in the level of performance is great. Since the full twist is introduced by many coaches in a manner that encourages contact twist, it might be that novice gymnasts have greater contact contributions than those shown here. Whether this is true or not, there is a case for learning this skill using aerial techniques since these are the predominant techniques used by elite gymnasts.

\section{Acknowledgement}

The support provided by the International Olympic Committee Medical Commission, The Sports Council, British Gymnastics and Loughborough University is gratefully acknowledged. 


\section{References}

Al-Haroun, M.R. (1980). Three dimensional cinematographic analysis of selected full twisting movements in gymnastics. Unpublished doctoral dissertation, Indiana University.

Batterman, C. (1974). The Techniques of Springboard Diving, $3^{\text {rd }}$ ed. Cambridge: MIT.

Boone, T. (1974). Biomechanics of a full twisting back salto. Scholastic Coach, 44, 3, 84-86.

Dowdell, P. (1988). Twisting tips. Gymnastic Coach, 7, 4, 28-31.

Frederick, A.B. (1971). The righting reflex of the cat. Journal of Health, Physical Education and Recreation, 42, 97-99.

Frolich, C. (1979). Do springboard divers violate angular momentum conservation? American Journal of Physics, 47, 7, 583-592.

Karara, H.M. (1980). Non-metric cameras. In K.B. Atkinson (Ed.), Developments in close range cinematography 1 (pp. 63-80). London: Applied Science Publishers.

McDonald, D. (1960). How does a cat fall on its feet? New Scientist, 7, 189, 1647-1649.

McDonald, D. (1961). How does a man twist in the air? New Scientist, 10, 237, 501-503.

Moore, A.C. (1951). A cinematographical analysis of a full twisting backward somersault. Unpublished Master's dissertation, University of Illinios.

Sanders, R.H. (1995). Effect of ability on twisting techniques in forward somersaults on the trampoline. Journal of Applied Biomechanics, 11, 267-287.

Seidel, H.S. (1976). A comparative analysis of the layout back somersault and the full twisting back somersault in tumbling. Unpublished Master's dissertation, Texas A \& M University.

Smith, G. (1980). Basic twisting concepts for gymnastics. International Gymnast, 22, 10, TS10-TS13.

Smith, T. (1982). Gymnastics: A mechanical understanding, pp. 82-86. London: Hodder and Staunton.

Van Gheluwe, B. (1981). A biomechanical simulation model for airborne twist in backward somersaults. Journal of Human Movement Studies, 7, 1-22.

Van Gheluwe, B. and Duquet, W. (1977). A cinematographic evaluation of two twisting theories in the backward somersault. Journal of Human Movement Studies, 3, 5-20.

Wiley, J.F. (1964). A cinematographic and mechanical analysis of the backward somersault with double twist in tumbling. Unpublished Master's dissertation, Sacramento State College.

Wood, G.A. and Jennings, L.S. (1979). On the use of spline functions for data smoothing. Journal of Biomechanics, 12, 477-479.

Yeadon, M.R. (1990a). The simulation of aerial movement - I: The determination of orientation angles from film data. Journal of Biomechanics, 23, 59-66.

Yeadon, M.R. (1990b). The simulation of aerial movement - II: A mathematical inertia model of the human body. Journal of Biomechanics, 23, 67-74. 
Yeadon, M.R. (1990c). The simulation of aerial movement - III: The determination of the angular momentum of the human body. Journal of Biomechanics, 23, 75-83.

Yeadon, M.R. (1993a). The biomechanics of twisting somersaults. Part I: Rigid body motions. Journal of Sports Sciences, 11, 187-198.

Yeadon, M.R. (1993b). The biomechanics of twisting somersaults. Part II: Contact twist. Journal of Sports Sciences, 11, 199-208.

Yeadon, M.R. (1993c). The biomechanics of twisting somersaults. Part III: Aerial twist. Journal of Sports Sciences, 11, 209-218.

Yeadon, M.R. (1993d). The biomechanics of twisting somersaults. Part IV: Partitioning performance using the tilt angle. Journal of Sports Sciences, 11, 219-225.

Yeadon, M.R. (1993e). Utilising nutation in twisting somersaults. In Proceedings of the Fourth International Symposium on Computer Simulation in Biomechanics (BMS3-16, 17). Paris: Ecole Nationale Superiere d'Arts et Metiers.

Yeadon, M.R. (1994). Twisting techniques used in high bar dismounts from the rings. Journal of Applied Biomechanics, 10, 178-188.

Yeadon, M.R. (1997). Twisting double somersault high bar dismounts. Journal of Applied Biomechanics, $13,76-87$.

Yeadon, M.R., Atha, J. and Hales, F.D. (1990a). The simulation of aerial movement - IV: A computer simulation model. Journal of Biomechanics, 23, 85-89.

Yeadon, M.R., Lee, S. and Kerwin, D.G. (1990b). Twisting techniques used in high bar dismounts. International Journal of Sport Biomechanics, 6, 139-146. 